\title{
Assessment of sunflower genotype tolerance to Phoma macdonaldii
}

\author{
Carine LARFEIL \\ Gérard BARRAULT \\ Grégory DECHAMP-GUILLAUME
}

ENSAT, UMR AGIR, BP 52627,

F-31326 Castanet-Tolosan cedex,

France

$<$ dechamp@ensat.fr>

\begin{abstract}
Sunflower genotypes with differing susceptibility to Phoma macdonaldii, the causal agent of sunflower black stem disease, were inoculated artificially with an aggressive fungal strain according to various modalities. A first series of experiments carried out in a greenhouse on plants at various phenological stages (2-leaf pairs, 5-6 leaf pairs, budding and early flowering) showed that the behaviour of sunflower cultivars, distributed into different susceptibility groups, remained unchanged irrespective of the phenological stage reached at the time of the inoculation. The development of the symptoms varied with genotype susceptibility to the disease: it was fast with coalescing necrotic spots on the stems for the most susceptible cultivars. The intensity and the development rate of the symptoms were also increased when the inoculation had been carried out at later stages. Two additional series of experiments were carried out in the field. In each series, a first block accounted for natural contaminations and a second block was inoculated artificially. The development of the symptoms and the susceptibility groups within the two blocks were identical to those observed in the greenhouse. These results led to the validation of an early inoculation test, at the cotyledon stage, which accounts satisfactorily for the behaviour of sunflower cultivars under natural conditions and is thus likely to be used in breeding programs.
\end{abstract}

Key words: black stem, Helianthus annuus, artificial inoculation, phenological stages, cultivar assessment

Phoma macdonaldii, the causal agent of sunflower black stem disease, has given rise to growing concern (Penaud and Pérès, 1995). The disease was called "Disease of the year" in 1992 as a result of its steady progression in France (Anonymous, 1998). The pathogen has been reported in various European countries [Yugoslavia, Bulgaria, Hungary, Romania (Maric et al., 1988), Asia (Iran (MadjidiehChassemi, 1988), Pakistan (Siddique-Mirza et al., 1988), China (Hua and Ma, 1996), the Americas (USA) (Acimovic, 1984; Donald et al., 1986), South America (Maric et al., 1988) and Australia (Acimovic, 1984; Miric et al., 1999)]. The occurrence of the fungal agent results in yield losses of $10-50 \%$ and reductions in oil content of the seeds and thousand-seed weight (Carson, 1991). Infection can occur at all phenological stages and on all sunflower organs. For the time being, as no sunflower cultivar is known to be resistant to Phoma, it would be interesting for plant breeders to use a reliable method for testing plant material within the framework of screening programs for sunflower resistance.

The aim of the investigations presented below was first to assess whether there are differences in the behaviour of sunflower cultivars in relation to their developmental stage. The absence of any kind of interaction is required for the possible development of a reliable early inoculation test. A first series of experiments was thus carried out in the greenhouse.
In a following step, the purpose of the experiments carried out in the field was to check whether the cultivar evaluation under controlled conditions was representative of the phenomena actually involved under natural conditions. The involvement of environmental factors in the development of the disease is not to be neglected since they might influence the behaviour of the genotypes in the presence of the disease. The inoculation experiments in the field were carried out during two successive campaigns. The results were then used for the possible validation of the early inoculation test developed in the laboratory and for an increased knowledge of disease development in relation to cultivar susceptibility and plant phenological stage.

\section{Materials and methods}

\section{Fungal isolate}

The aggressive strain T32A142 originating from Southwestern France was selected for investigations.

\section{Plant material}

Seven sunflower cultivars with differing susceptibility to Phoma were investigated: DK3825 (RAGT), Santiago (Novartis Seeds SA), Andora (Pau Semences), Argos (Verneuil), Heliasol (Semences de France), Melody (Novartis Seeds SA) and Prodisol (Dekalb). The seed companies communicated information issued from field observations and the behaviour of the cultivars was tested in the laboratory.

\section{Greenhouse experiments at various phenological stages}

The various phenological stages selected for the investigations were 2-leaf pairs, 4-6 leaf pairs, budding and early flowering. Sunflower hybrids DK3825, Santiago, Andora, Argos, Heliasol and Prodisol were then used.

For the 2-leaf pair stage, the seeds were sown uniformly at a depth of $2 \mathrm{~cm}$ in $40 \times 30 \times 25 \mathrm{~cm}$ plastic containers filled with a peat moss-clay mixture (Florathon ${ }^{\circledR} 2$, F-Wolsheim, France). Two containers were used for each cultivar, with 15 seeds per container. For the other phenological stages, the seeds were sown in pots, using 10 pots for each cultivar, with one seed per pot.

The plants were developed in the greenhouse until the selected phenological stage was reached: 14 days for the 2-leaf pair stage, 4 weeks for the 5-6 leaf pair stage, 5 weeks for the budding stage and 8 weeks for the early flowering stage.

\section{Field experiments}

Two series of experiments were carried out in 2000 and in 2002, respectively. In each case, plots of the CETIOM En-Crambade (HauteGaronne) Agricultural Experiment Station were used. Each sunflower hybrid was sown on 510 m-long rows using 35 seeds per row. The distance between the rows was $53 \mathrm{~cm}$. 
No chemical treatment was applied to the plants. The soil was watered 24 hours prior to inoculation (the water supply was equivalent to 10-mm rainfall).

For the 2000 campaign, the 5 sunflower cultivars sown were Prodisol, Andora, Argos, Santiago and DK3825. For each hybrid, only one of the 5 rows was inoculated artificially; the other rows were used as controls with possible natural contamination.

For the 2002 campaign, in addition to the 5 genotypes already mentioned, Melody and Heliasol were also selected, taking into account the behaviour observed in a phytotron. Melody is a tolerant cultivar, whereas Heliasol is very susceptible to Phoma attacks. The experimental design was the same as that mentioned for the 2000 campaign.

\section{Inoculation and symptom assessment}

\section{Greenhouse experiments at various phenological stages}

At the 2-leaf pair stage, the seedlings were inoculated on the cotyledon petioles, using the method described by Roustaee et al. (2000). The assessment of symptom severity was based on the percent of necrotic area on the cotyledon petiole, using a 1-9 scale (table 1).

In the other trials, the plants were inoculated at the insertion point of the petiole of the 3-leaf pairs located above leaf pair 2. The amounts of inoculum were $50 \mu \mathrm{L}$ for the 5-6 leaf pair stage, $80 \mu \mathrm{L}$ for the budding stage and $100 \mu \mathrm{L}$ for the early flowering stage. The inoculation sites were identified using a felt pen. The moisture was maintained for 48 hours by using plastic bags covering the plants and placing water-filled trays under the containers. The development of the symptoms was recorded for 6 weeks, with one observation per week.

Table 1. Scale based on the percent of necrotic cotyledon petiole after inoculation with Phoma macdonaldii.

\begin{tabular}{|lc|}
\hline Score & \% necrotic petiole \\
\hline 0 & 0 \\
\hline 1 & $0-5$ \\
\hline 2 & $5-10$ \\
\hline 3 & $10-20$ \\
\hline 4 & $20-30$ \\
\hline 5 & $30-40$ \\
\hline 6 & $40-60$ \\
\hline 7 & $60-80$ \\
\hline 8 & $80-100$ \\
\hline 9 & 100 \\
\hline
\end{tabular}

Table 2. Scale based on necrotic stem length.

\begin{tabular}{|ll|}
\hline Score & Necrotic stem length \\
\hline 1 & $<1.5 \mathrm{~cm}$ \\
\hline 2 & $1.5-3 \mathrm{~cm}$ \\
\hline 3 & $3-6 \mathrm{~cm}$ \\
\hline 4 & $>6 \mathrm{~cm}$ \\
\hline
\end{tabular}

The scale with four levels was based on the length of the necrotic area on the stem (table 2).

\section{Field experiments}

The inoculation was carried out at the 5-6 leaf pair stage through the addition of $100 \mu$ of a pycniospore suspension at the insertion point of the petiole on the stem. Three levels corresponding to three leaf pairs were thus inoculated, the inoculation starting from leaf pair 3. Rings were used for the recognition of the inoculated petioles.

The plants were then maintained under saturating humidity for 48 hours using a plastic tunnel. For the 2000 campaign, the development of the symptoms was followed for 8 weeks, with one observation per week, using a scale based on the length of the necrotic area on the stem (table 2). For the 2002 campaign, only one observation was carried out 8 weeks after the inoculation so as to corroborate the earlier observations (2000 campaign) and obtain an assessment of the cultivars. The same scale as above was then used.

\section{Data analyses}

The data were submitted to variance analyses and the means were compared using NewmanKeuls test $(P=0.05)$. No transformation was required for the standardization of the distributions. For the greenhouse experiments, the data taken into account for variance analyses were the observations carried out 6 weeks after inoculation for all stages, with the exception of the 2-leaf pair stage (7 days after inoculation). For the field experiments, the observations carried out 8 weeks after inoculation were taken into account.

\section{Results}

\section{Greenhouse experiments at various phenological stages}

\section{Assessment of genotype tolerance}

\section{2-leaf pair stage}

A classification of the genotypes was established from the scores of the symptoms on the cotyledon petioles. Significant differences in behaviour were observed between the cultivars $(F=2.97 ; P<0.05)$. Prodisol and Heliasol were the most susceptible cultivars; DK3825 was the most tolerant, whereas the other genotypes were intermediate (table 3). The test was thus rather discriminating for a differentiation of the cultivars in their behaviour towards Phoma.

\section{5-6 leaf pair, budding and early flowering stages}

The results obtained for the three types of variance analyses carried out are presented in table 4.

The first variance analysis led to a classification of sunflower genotypes in relation to their susceptibility to Phoma at each phenological stage. The means are to be read vertically and the homogeneous groups obtained are defined by the letters to the left of the means. For each phenological stage, significant differences in behaviour were observed between the cultivars ( $F$ genotypes $=3.91^{* *}, P<0.01$ for the 5-6 leaf pair stage; $F$ genotypes = $7.63^{* * *}, P<0.001$ for the budding stage; $F$ genotypes $=7.17^{* \star *}, P<0.001$ for the early flowering stage). However, for the 5-6 leaf pair stage, the sunflower cultivars belonged to the same homogeneous group, whereas the differences in behaviour between genotypes were visually outstanding. This discrepancy is to be assigned to the four-level scale used: each level corresponds to large differences in lengths of necrotic areas whereas, statistically, the differences are small. Irrespective of the phenological stage considered, the classification of the sunflower cultivars is globally the same. Prodisol is the most susceptible genotype, irrespective of the developmental stage. Argos, Andora and Heliasol are moderately susceptible varieties and their rank within this susceptibility group may change in relation to the phenological stage. DK3825 was the most tolerant cultivar in most cases, whereas Santiago was tolerant or moderately susceptible, depending on the developmental stage. The very susceptible cultivar Prodisol was thoroughly necrotic, whereas the tolerant varieties

Table 3. Classification of 6 sunflower cultivars inoculated with strain T32A142 at the 2-leaf pair stage, scores after 7 days in the greenhouse.

\begin{tabular}{|lll|}
\hline Genotypes & Score & $\begin{array}{l}\text { Homogeneous } \\
\text { groups }\end{array}$ \\
\hline Prodisol & 8 & $\mathrm{~A}$ \\
\hline Heliasol & 7.77 & $\mathrm{~A}$ \\
\hline Andora & 7.41 & $\mathrm{~A} \mathrm{~B}$ \\
\hline Santiago & 7.23 & $\mathrm{~A} \mathrm{~B}$ \\
\hline Argos & 6.59 & $\mathrm{~A} \mathrm{~B}$ \\
\hline DK3825 & 6.09 & $\mathrm{~B}$ \\
\hline
\end{tabular}

Fgenotypes $=2.97^{*}, P<0.01$. 
Table 4. Classification of 6 sunflower cultivars inoculated with strain T32A142 at various phenological stages, scores after 6 weeks in the greenhouse.

\begin{tabular}{|c|c|c|c|c|}
\hline Genotypes & 5-6 leaf pair stage & Budding stage & Early flowering stage & $\begin{array}{l}\text { Means } \\
\text { Homogeneous groups }\end{array}$ \\
\hline Prodisol & a2.47c & $a 3.29 b$ & a3.95a & $3.23 \mathrm{~A}$ \\
\hline Argos & $a 2.31 b$ & ab3.08a & b2.96a & $2.78 B$ \\
\hline Andora & $\mathrm{a} 1.87 \mathrm{~b}$ & ab2.79a & b2.94a & $2.53 B$ \\
\hline Heliasol & a2.44ns & ab2.5ns & b2.44ns & $2.46 B$ \\
\hline DK3825 & a1.55b & b2.38a & b2.31a & $2.08 \mathrm{C}$ \\
\hline Santiago & a1.56b & $c 1.60 b$ & b2.54a & $1.90 \mathrm{C}$ \\
\hline Means & 2.03 & 2.60 & 2.86 & \\
\hline Homogeneous groups & $\mathrm{C}$ & B & A & \\
\hline
\end{tabular}

$F$ stages $=22.3^{* * *}, P<0.001 ; F$ genotypes $=14.53^{* * *}, P<0.001 ; F$ stages $\times$ genotypes $=0.29 \mathrm{~ns}$. Means with the same letter do not differ significantly. Letters to the left of each value refer to differences between cultivars for a given stage. Letters to the right of each value refer to differences between stages for a given cultivar. n.s.: not significant.

DK3825 and Santiago displayed only a few necrotic spots at the inoculation point without subsequent development. The other genotypes were characterized by an intermediate behaviour with wider necrotic areas than in tolerant varieties. More generally, the more susceptible and the more tolerant genotypes kept their behaviour towards the disease, irrespective of the phenological stage.

The second variance analysis led to the determination of the behaviour of each of the 6 cultivars towards Phoma in relation to the phenological stage. The means are to be read horizontally, and the homogeneous groups obtained are defined by the letters to the right of the means. For each cultivar, with the exception of Heliasol, significant differences in behaviour were observed in relation to the developmental stage $\left(F\right.$ stages $=5.84^{*}$, $P<0.05$ for Andora; $F$ stages $=5.48^{*}$, $P<0.05$ for Argos; $F$ stages $=6.21^{* *}, P<0.01$ for DK3825; F stages $=13.45^{* * *}, P<0.001$ for Prodisol; $F$ stages $=5.59^{*}, \quad P<0.05$ for Santiago). The behaviour of the genotypes was nearly the same at the budding and early flowering stages, with the exception of Prodisol which displayed a different reaction at each stage and of Santiago whose behaviour was identical for the two youngest stages.

The third variance analysis showed there was no interaction between the phenological stage and the behaviour of the genotypes towards the disease and led to the characterization of the differences between the phenological stages independently of the cultivars, as well as between the genotypes independently of the developmental stages. There were significant differences between the stages $(F=22.3, P<0.001)$ and between the cultivars $(F=14.53, P<0.001)$. Thus, the genotypes could be grouped into 3 different susceptibility groups which are the same as those presented earlier. Prodisol was the most susceptible cultivar and DK3825 the most tolerant. Santiago was rather tolerant, whereas Heliasol, Argos and Andora were moderately susceptible. The intensity of the symptoms depended on the phenological stage considered. As the inoculation occurred at an advanced stage, the symptoms were more severe. As there was no interaction between the phenological stage and the cultivar factors, the genotype behaviour was identical irrespective of the phenological stage, although the symptoms were more severe from the budding stage.

\section{Disease kinetics}

The development of the disease was represented taking into account only the percentage of levels 3 and 4 on the scale used for the severity of the disease (1-100\% scale), as all the genotypes can thus be compared to the extent that the corresponding lengths of necrotic area are present on the tolerant cultivars.
Plant developmental stage had a high incidence on disease severity. At the 5-6 leaf pair stage, the extension and the expression of the symptoms were much lower (figure 1). The appearance of necroses on the stems occurred mostly 3 weeks after the inoculation (severity 5\%), and the development of the symptoms was quite slow (severity in the range 3.3-33\% after 5 weeks). At the budding stage, the symptoms on the stems were hardly present 2 weeks after inoculation; they appeared mostly on week 3 (severity $1.67-8.3 \%$ ) or even on week 4 after the inoculation, depending on the genotypes (figure 2). The development of the symptoms was however rapid though late (severity 8.3-62.8\% after 5 weeks). At the early flowering stage, the development of the symptoms was quite fast for all genotypes (figure 3). Large necroses appeared as soon as week 2 (severity $1.7-13 \%)$ and extended steadily until week 5 (severity 11.7-96\%, depending on the genotypes).

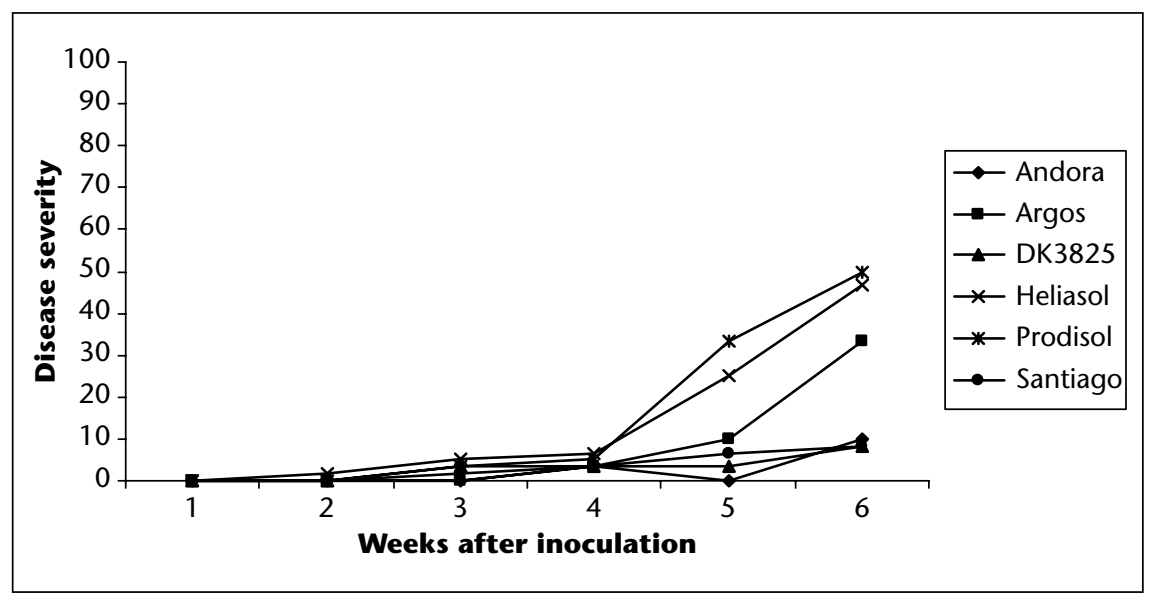

Figure 1. Symptom development in 6 sunflower cultivars inoculated with strain T32A142 at the 5-6 leaf pair stage in the greenhouse. 


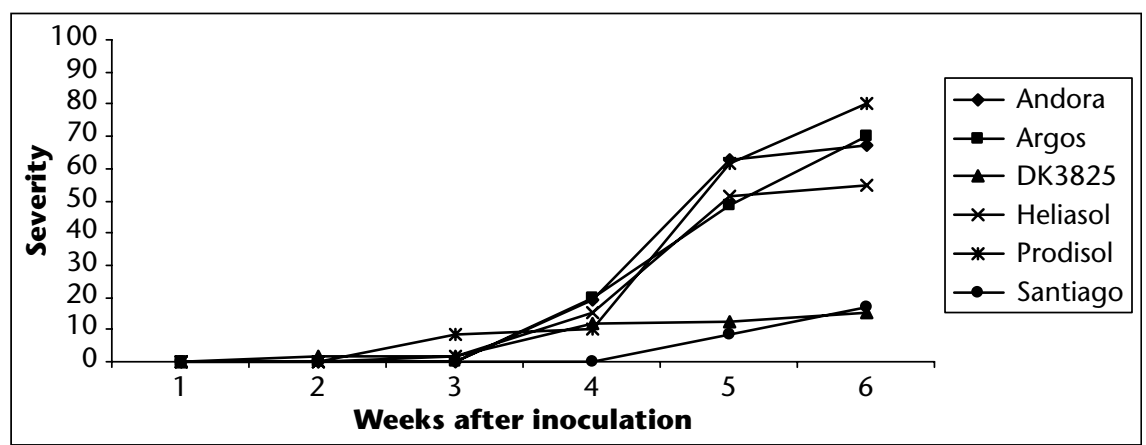

Figure 2. Symptom development in 6 sunflower cultivars inoculated with strain T32A142 at the budding stage in the greenhouse.

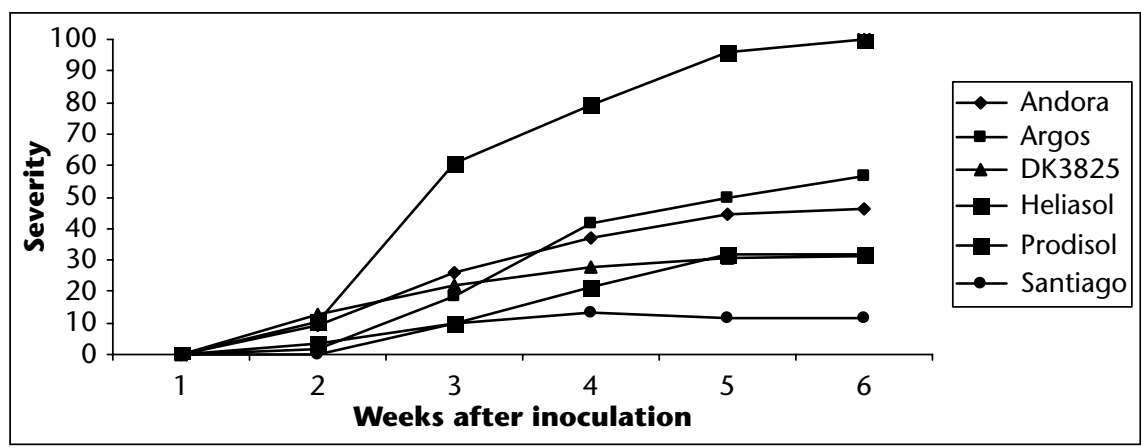

Figure 3. Symptom development in 6 sunflower cultivars inoculated with strain T32A142 at the early flowering stage in the greenhouse.
Disease kinetics also differed with genotype susceptibility. In Prodisol, a very susceptible cultivar, the development of the symptoms was quite fast, especially at the early flowering stage, with the very early appearance of cracked block sleeves and the attack of the pith. Moderately susceptible cultivars such as Andora, Argos and Heliasol were characterized by a gradual, though intense, development of the symptoms (severity $55-70 \%$ for the budding stage 6 weeks after the inoculation). In some cases, tissue hypertrophy was observed. In the tolerant genotypes, the development of the symptoms was very slow (severity $10-30 \%$ 6 weeks after the inoculation, irrespective of the phenological stage).

\section{Field experiments}

\section{Campaign 2000}

Variance analyses showed the occurrence of significant differences in behaviour between genotypes in response to an attack by Phoma macdonaldii in the field ( $F$ genotypes $=44.69$, $P<0.001$ ). The cultivars can be classed into 3 susceptibility groups. Prodisol isvery susceptible, Andora, Argos and Santiago are moderately susceptible and DK3825 is tolerant (table 5).

On the diseased plants, the appearance of the symptoms occurred as soon as the first week.
Table 5. Classification of 5 sunflower cultivars inoculated with strain T32A142 at the 5-6 leaf pair stage in the field, scores after 8 weeks - campaign 2000.

\begin{tabular}{|lll|}
\hline Genotypes & Score & $\begin{array}{l}\text { Homogeneous } \\
\text { groups }\end{array}$ \\
\hline Prodisol & 3.80 & $\mathrm{~A}$ \\
\hline Andora & 3.07 & $\mathrm{~B}$ \\
\hline Santiago & 2.95 & $\mathrm{~B}$ \\
\hline Argos & 2.93 & $\mathrm{~B}$ \\
\hline DK3825 & 2.41 & $\mathrm{C}$ \\
\hline
\end{tabular}

Fgenotypes $=44.69^{* * *}, P<0.001$.

stem with the development of necrotic spots in length and width. In some cases, the coalescence of the necroses gave rise to sleeves surrounding the stem.

A natural Phoma attack was observed on control plants with identical symptoms to those already described. The natural attack occurred in mid-June, 3 weeks after the artificial inoculation. The development and the intensity of the symptoms due to natural contaminations were comparable to those of artificial contaminations. The classification of the sunflower genotypes according to their susceptibility to Phoma is the same: Prodisol is very susceptible, DK3825 is tolerant and the other genotypes display an intermediate susceptibility.

Black necroses developed on the petiole, and then rapidly extended to the whole petiole length, with, in some cases, the formation of characteristic "goosefoot" symptoms. The disease then proceeded further, invading the

The development of the disease on the 5 sunflower genotypes investigated is shown in figure 4 and table 6. Disease kinetics varied in relation to genotype susceptibility. In the

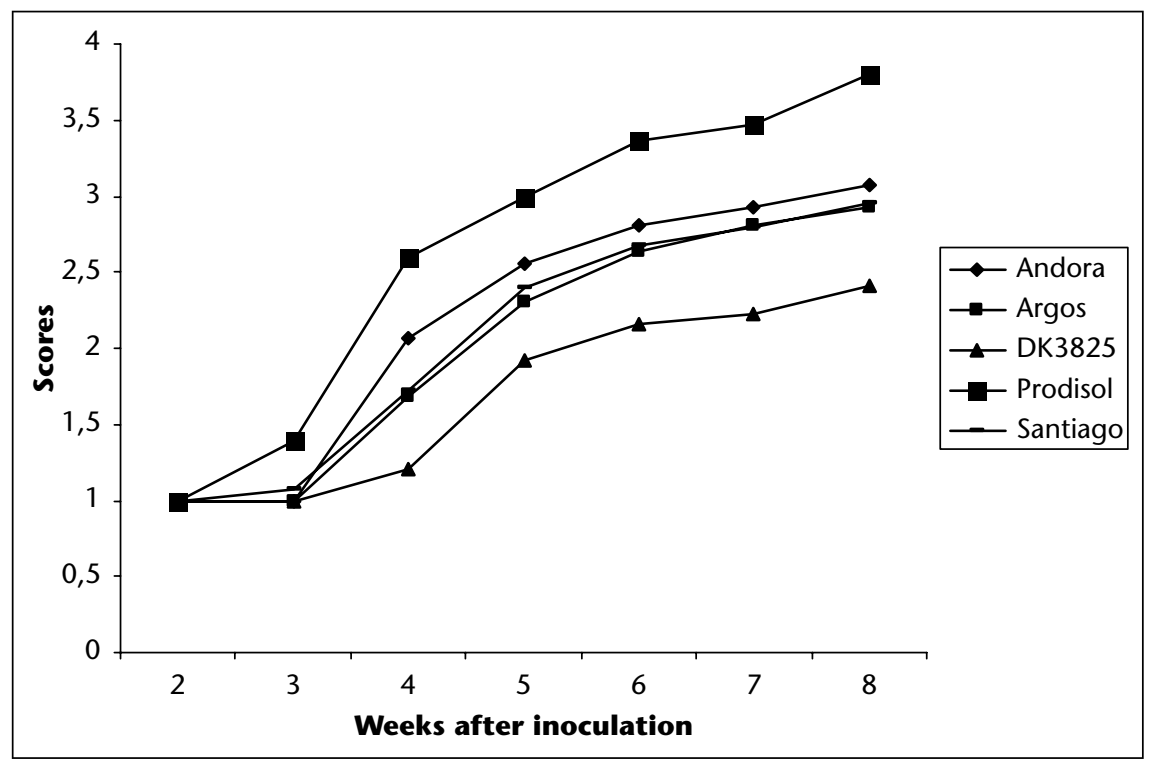

Figure 4. Symptom development in 5 sunflower cultivars inoculated with strain T32A142 at the 5-6 leaf pair stage in the field, campaign 2000. 
Table 6. Development of disease symptoms on 5 sunflower cultivars inoculated with strain T32A142 at the 5-6 leaf pair stage in the field, scores after 7 weeks - campaign 2000.

\begin{tabular}{|c|c|c|c|c|c|c|c|}
\hline \multirow[b]{2}{*}{ Cultivars } & \multicolumn{7}{|c|}{ Weeks after inoculation } \\
\hline & 2 & 3 & 4 & 5 & 6 & 7 & 8 \\
\hline Andora & $1 \pm 0$ & $1 \pm 0$ & $2.07 \pm 0.43$ & $2.55 \pm 0.37$ & $2.81 \pm 0.27$ & $2.93 \pm 0.32$ & $3.07 \pm 0.35$ \\
\hline Argos & $1 \pm 0$ & $1 \pm 0$ & $1.68 \pm 0.51$ & $2.3 \pm 0.35$ & $2.64 \pm 0.25$ & $2.81 \pm 0.3$ & $2.93 \pm 0.6$ \\
\hline DK3825 & $1 \pm 0$ & $1 \pm 0$ & $1.21 \pm 0.37$ & $1.92 \pm 0.61$ & $2.16 \pm 0.56$ & $2.23 \pm 0.53$ & $2.41 \pm 0.45$ \\
\hline Prodisol & $1 \pm 0$ & $1.39 \pm 0.43$ & $2.59 \pm 0.46$ & $3 \pm 0.33$ & $3.37 \pm 0.3$ & $3.47 \pm 0.33$ & $3.8 \pm 0.37$ \\
\hline Santiago & $1 \pm 0$ & $1.07 \pm 0.17$ & $1.72 \pm 0.51$ & $2.4 \pm 0.47$ & $2.68 \pm 0.34$ & $2.8 \pm 0.32$ & $2.95 \pm 0.26$ \\
\hline
\end{tabular}

susceptible cultivar Prodisol, the development of the symptoms was quite rapid, right from the starting stages. On week 7, the stems were thoroughly necrotic. In the tolerant cultivar DK3825, the development of the disease was quite slow, with a low extension of the necroses. In the moderately susceptible cultivars, Andora, Argos and Santiago, the development of the symptoms was intermediate between those described for the susceptible and tolerant genotypes.

\section{Campaign 2002}

As a result of a high increase in temperature (up to $40{ }^{\circ} \mathrm{C}$ or even more) on day 2 , some genotypes were submitted to a severe desiccation which was lethal in some cases. A second inoculation carried out 2 weeks later in the absence of the plastic tunnel (due to plant height) failed, which emphasized the role of saturating humidity during the inoculation phase. As a natural infection had developed on control plants, the data issued from the observations of the symptoms on 7 genotypes could be processed, since the genotype behaviour is the same, whether the inoculation is natural or artificial (table 7).

Prodisol is a very susceptible sunflower cultivar, whereas DK3825 is the most tolerant. Andora,

Table 7. Classification of 7 sunflower cultivars inoculated with strain T32A142 at the 5-6 leaf pair stage in the field, scores after 8 weeks - campaign 2002.

\begin{tabular}{|lll|}
\hline Genotypes & Score & $\begin{array}{l}\text { Homogeneous } \\
\text { groups }\end{array}$ \\
\hline Prodisol & 3.20 & $\mathrm{~A}$ \\
\hline Heliasol & 2.87 & $\mathrm{~B}$ \\
\hline Melody & 2.70 & $\mathrm{~B}$ \\
\hline Argos & 2.37 & $\mathrm{C}$ \\
\hline Santiago & 2.20 & $\mathrm{CD}$ \\
\hline Andora & 2.03 & $\mathrm{CD}$ \\
\hline DK3825 & 1.93 & $\mathrm{D}$ \\
\hline
\end{tabular}

Fgenotypes $=17.17^{* * *}, P<0.001$
Santiago and Argos, with an intermediate behaviour, are moderately susceptible to Phoma, whereas Melody, which is close to Heliasol, appeared as a rather susceptible cultivar. In Prodisol, a very susceptible genotype, the necroses were readily coalescent. In the most tolerant genotype DK3825, the development of the necrotic spots was nearly restricted to the inoculation point. In sunflower hybrid Argos, a genotype of intermediate susceptibility, the necrotic spots developed without forming sleeves. The classification thus obtained corroborated that observed in the field for campaign 2000. The susceptible behaviour of Heliasol is consistent with earlier observations in the greenhouse. In contrast, Melody, which seemed rather tolerant in the phytotron (at the seedling stage), was rather susceptible in the field. However, preliminary investigations with strain MP6 suggested the possible occurrence of differences in behaviour for some genotypes according to the fungal strain used. It is noteworthy that the genotypes with an extreme behaviour were satisfactorily discriminated in these field experiments. Tolerant genotypes such as DK3825 remained tolerant in the field and the most susceptible genotype Prodisol remained very susceptible to the disease. A satisfactory discrimination between susceptible, very susceptible and tolerant genotypes is thus essential.

\section{Discussion}

The greenhouse experiments led to a better understanding of disease development in relation to genotype susceptibility and phenological stage.

Irrespective of the genotypes, the infection starts with the appearance of necroses which develop more or less rapidly according to the susceptibility of the cultivar. In very susceptible genotypes, the extension of the necroses is very rapid whereas, in tolerant genotypes, little development of the necroses occurs. Fayzalla and Maric (1981) had already observed such phenomena in relation to the developmental stage of the host plant: the susceptibility to Phoma was different, the most sensitive stage being flowering. Irrespective of the plant developmental stage, the behaviour of the cultivars is identical: the absence of interaction is essential and quite valuable for the implementation of cultivar assessment tests. The early inoculation test on cotyledon petioles leads to a satisfactory discrimination of the genotypes with a high susceptibility to Phoma and is also consistent with the classification of the genotypes which can be established at the other phenological stages. The artificial inoculation of leaf petioles with Phoma lingam pycniospores proved to be efficient in rape cultivar screening (Brunin and Lacoste, 1970; Newman and Bailey, 1987) and in other crucifers (Williams and Delwiche, 1979). In Phoma exigua, the effect observed is the opposite of that reported for Phoma macdonaldii: the early stages (seed and cotyledon) are much more susceptible to the disease than later stages such as flowering (Decognet, 1994).

The results observed in the field were comparable to those obtained in the greenhouse at various phenological stages. In both cases, DK3825 was the most tolerant genotype, Prodisol the most susceptible and the other genotypes displaying an intermediate susceptibility. From campaign 2000 to campaign 2002, even though genotype assessment was similar, disease severity was lower during the latter campaign. Lower temperatures throughout campaign 2002 can account for the relative decrease in disease severity, illustrating the decisive effect of environmental factors, and more particularly of climatic conditions, on disease development. The determining influences of moisture and temperature were mentioned: these essential factors condition the success of the inoculation. The higher or lower incidence of Phoma disease according to the years and the regions is related to the occurrence of more or less favourable environmental conditions to the infection and development of the disease. The decisive role of moisture had also been demonstrated in Phoma exigua: the 
fungal attack is conditioned by soil moisture as well as by atmospheric humidity, which have to be above $50 \%$ for a successful initiation of the infection (Decognet, 1994).

The results detailed above show that the early cultivar assessment test is quite reliable since there are no bias due to the host phenological stage and since very susceptible, susceptible and tolerant genotypes can thus be discriminated satisfactorily. Moreover, the contamination level at the 2-leaf pair stage affords a prediction of the behaviour of the genotype in the field, particularly if the susceptibility level of the genotype is quite high or quite low. The classification obtained for the genotypes is consistent with the data already known for each cultivar. This inoculation method might thus be useful and valuable for genotype evaluation tests.

Acknowledgements. We would like to thank research centres and farmers who supported or undertook the trials: CETIOM Grignon and CETIOM En-Crambade. ONIOL provided some support for this programme.

\section{REFERENCES}

Acimovic MA. Sunflower diseases in Europe, the United States, and Australia, 1981-1983. Helia 1984; 7: 45-54.
Anonymous. Actualités semences Tournesol. Semences et Progrès 1998; 94: 62-8.

Brunin B, Lacoste L. Recherches sur la maladie du colza due à Leptosphaeria maculans (desm.); pouvoir pathogène des ascospores. Annales de Phytopathologie 1970; 2: 477-88.

Carson ML. Relationship between Phoma black stem severity and yield losses in hybrid sunflower. Plant Disease 1991; 75: 1150-3.

Decognet V. Phoma exigua var. linicola, agent du mort-lin: variabilité et mode d'infection du parasite, expression de la maladie. Ph.D. thesis, Université de Rennes (France) 1994; 112 p.

Donald PA, Bugbee WM, Venette JR. First report of Leptosphaeria lindquistii (sexual stage of Phoma macdonaldii) on sunflower in North Dakota and Minnesota. Plant Disease 1986; 70: 352.

Fayzalla S, Maric A. Contribution à l'étude de la biologie et de l'épidémiologie de Phoma macdonaldii Boerema provoquant la maladie des taches noires du tournesol. Zastita Bilja 1981; 32: 13-27.

Hua Z, Ma G. A review of sunflower disease research in China. In: Proceedings of the Fourteenth International Sunflower Conference, Beijing (China) 1996 ; 754-9.

Madjidieh-Ghassemi S. Studies of some important fungal diseases of sunflower in Iran. In: Proceedings of the Twelfth International Sunflower Conference, Novi Sad (Yugoslavia) $1988 ; 2: 22-3$.
Maric A, Camprag D, Magirevic S. La tacheture noire du tournesol (Phoma macdonaldii Boerema; synonymes: Phoma oleracea var. helianthi-tuberosi Sacc. stade terminal: Leptosphaeria lindquisti Frezzi). Bolesti i stetocine suncokretai njihovo suzbijanje 1988 : 37-45.

Miric E, Aitken EAB, Goulter KC. Identification in Australia of the quarantine pathogen of sunflower Phoma macdonaldii (Teleomorph: Leptosphaeria linquistii). Australian Journal of Agricultural Research 1999; 50: 325-32.

Newman PL, Bailey DJ. Screening for resistance to canker (Leptosphaeria maculans) in winter oilseed rape (Brassica napus spp. oleifera). Plant Pathology 1987; 36: 346-54.

Penaud A, Pérès A. Phoma du tournesol. Oléoscope $1995 ; 15: 37 \mathrm{p}$.

Roustaee A, Costes S, Dechamp-Guillaume G, Barrault G. Phenotypic variability of Leptosphaeria lindquistii (anamorph: Phoma macdonaldii), a fungal pathogen of sunflower. Plant Pathology 2000; 49: 227-34.

Siddique-Mirza M, Masood AR, Ayub M. Sunflower diseases in Pakistan in the period 1980-87. In: Proceedings of the Twelfth International Sunflower Conference, Novi Sad (Yugoslavia) $1988 ; 2: 25$.

Williams PH, Delwiche PA. Screenings for resistance to blackleg of crucifers in the seedling stage. In: Proceedings of Eucarpia Conference, Wageningen (The Netherlands) $1979 ; 164-170$. 